University of Wollongong

Research Online

Australian Institute for Innovative Materials -

Papers

Australian Institute for Innovative Materials

$1-1-2014$

Converse flexoelectric coefficient $\mathrm{f}(1212)$ in bulk Ba0.67Sr0.33TiO3

Longlong Shu

North Carolina State University

Wenbin Huang

North Carolina State University

Seol Ryung Kwon

North Carolina State University

Zhao Wang

Xi'an Jiaotong University

Fei Li

Xi'an Jiaotong University, lifei1216@gmail.com

See next page for additional authors

Follow this and additional works at: https://ro.uow.edu.au/aiimpapers

Part of the Engineering Commons, and the Physical Sciences and Mathematics Commons

Research Online is the open access institutional repository for the University of Wollongong. For further information contact the UOW Library: research-pubs@uow.edu.au 


\title{
Converse flexoelectric coefficient $f(1212)$ in bulk Ba0.67Sr0.33TiO3
}

\author{
Abstract \\ Enhanced flexoelectricity in perovskite ceramics and single crystals has been reported before. In this \\ letter, 3-3 ceramic-ceramic $\mathrm{Ba} 0.6 \mathrm{Sr} 0.4 \mathrm{TiO} / \mathrm{Ni0} .8 \mathrm{Zn} 0.2 \mathrm{Fe} 2 \mathrm{O} 4$ composite with a colossal permittivity was \\ employed in the conventional pure bending experiment in order to examine the transverse flexoelectric \\ response. The measured flexoelectric coefficient at $30 \mathrm{~Hz}$ is $128 \mu \mathrm{C} / \mathrm{m}$ and varies to $16 \mu \mathrm{C} / \mathrm{m}$ with the \\ frequency increasing from $30 \mathrm{~Hz}$ to $120 \mathrm{~Hz}$, mainly due to the inverse correlation between the permittivity \\ and the frequency. This result reveals the permittivity dependence of flexoelectric coefficient in the \\ frequency dispersion materials, suggesting that the giant permittivity composites can be good \\ flexoelectric materials. \\ Disciplines \\ Engineering | Physical Sciences and Mathematics

\section{Publication Details} \\ Shu, L., Huang, W., Kwon, S. Ryung., Wang, Z., Li, F., Wei, X., Zhang, S., Lanagan, M., Yao, X. \& Jiang, X. \\ (2014). Converse flexoelectric coefficient $\mathrm{f}(1212)$ in bulk Ba0.67Sr0.33Ti03. Applied Physics Letters, 104 \\ (23), 232902-1-232902-6.
}

\section{Authors}

Longlong Shu, Wenbin Huang, Seol Ryung Kwon, Zhao Wang, Fei Li, Xiaoyong Wei, Shujun Zhang, Michael T. Lanagan, Xi Yao, and Xiaoning Jiang 


\section{Converse flexoelectric coefficient $\mathrm{f}_{1212}$ in bulk $\mathrm{Ba}_{0.67} \mathrm{Sr}_{0.33} \mathrm{TiO}_{3}$}

Longlong Shu, Wenbin Huang, Seol Ryung Kwon, Zhao Wang, Fei Li, Xiaoyong Wei, Shujun Zhang, Michael Lanagan, Xi Yao, and Xiaoning Jiang

Citation: Appl. Phys. Lett. 104, 232902 (2014); doi: 10.1063/1.4882060

View online: https://doi.org/10.1063/1.4882060

View Table of Contents: http://aip.scitation.org/toc/apl/104/23

Published by the American Institute of Physics

\section{Articles you may be interested in}

Relationship between direct and converse flexoelectric coefficients

Journal of Applied Physics 116, 144105 (2014); 10.1063/1.4897647

Flexoelectricity in barium strontium titanate thin film

Applied Physics Letters 105, 142904 (2014); 10.1063/1.4898139

Experimental studies of the converse flexoelectric effect induced by inhomogeneous electric field in a barium strontium titanate composition

Journal of Applied Physics 100, 024112 (2006); 10.1063/1.2219990

Enhanced direct flexoelectricity in paraelectric phase of $\mathrm{Ba}\left(\mathrm{Ti}_{0.87} \mathrm{Sn}_{0.13}\right) \mathrm{O}_{3}$ ceramics

Applied Physics Letters 102, 152904 (2013); 10.1063/1.4802450

Frequency dispersion of flexoelectricity in PMN-PT single crystal

AIP Advances 7, 015010 (2017); 10.1063/1.4973684

Giant flexoelectricity in $\mathrm{Ba}_{0.6} \mathrm{Sr}_{0.4} \mathrm{TiO}_{3} / \mathrm{Ni}_{0.8} \mathrm{Zn}_{0.2} \mathrm{Fe}_{2} \mathrm{O}_{4}$ composite

Applied Physics Letters 105, 162906 (2014); 10.1063/1.4899060

\section{PHYSICS TODAY}

MANAGER'S GUIDE

WHITEPAPERS

\section{READ NOW}

PRESENTED BY

Accelerate R\&D with
Multiphysics Simulation 


\title{
Converse flexoelectric coefficient $f_{1212}$ in bulk $\mathrm{Ba}_{0.67} \mathrm{Sr}_{0.33} \mathrm{TiO}_{3}$
}

\author{
Longlong Shu, ${ }^{1,2}$ Wenbin Huang, ${ }^{1}$ Seol Ryung Kwon, ${ }^{1}$ Zhao Wang, ${ }^{2} \mathrm{Fei} \mathrm{Li}^{2}$ \\ Xiaoyong Wei, ${ }^{2, a)}$ Shujun Zhang, ${ }^{3}$ Michael Lanagan, ${ }^{3}$ Xi Yao, ${ }^{2}$ and Xiaoning Jiang ${ }^{1, b)}$ \\ ${ }^{1}$ Department of Mechanical and Aerospace Engineering, North Carolina State University, Raleigh, \\ North Carolina 27695, USA \\ ${ }^{2}$ Electronic Materials Research Laboratory, Key Laboratory of the Ministry of Education and International \\ Center for Dielectric Research, Xi' an Jiaotong University, Xi' an 710049, China \\ ${ }^{3}$ Materials Research Institute, Pennsylvania State University, University Park, Pennsylvania 16802, USA
}

(Received 16 April 2014; accepted 27 May 2014; published online 9 June 2014)

\begin{abstract}
The converse flexoelectric effect, referred as the electric field gradient induced strain, widely exists in dielectric materials, but its experimental studies have been reported by few research groups so far. In this Letter, we report our studies on the converse flexoelectric behavior of $\left(\mathrm{Ba}_{0.67} \mathrm{Sr}_{0.33}\right) \mathrm{TiO}_{3}$ ceramics and present the measured value of its flexoelectric coefficient $f_{1212}$. In the experiments, the electric field gradient was generated by applying an electric field across the two lateral sides of trapezoid $\left(\mathrm{Ba}_{0.67} \mathrm{Sr}_{0.33}\right) \mathrm{TiO}_{3}$ samples. The shear displacement was measured using a laser vibrometer. The converse flexoelectric coefficient $f_{1212}$ was found to be $124 \pm 14 \mu \mathrm{C} / \mathrm{m}$ at room temperature. This result was in good agreement with the theoretical prediction of the flexoelectricity of the $(\mathrm{Ba}, \mathrm{Sr}) \mathrm{TiO}_{3}$ ceramics. (C) 2014 AIP Publishing LLC.

[http://dx.doi.org/10.1063/1.4882060]
\end{abstract}

Flexoelectricity, denoting the electric polarization induced by mechanical strain gradient or mechanical strain induced by electric field gradient, has drawn research interests in recent years. ${ }^{1-3}$ This phenomenon is generally expressed in tensor form ${ }^{4}$

$$
\begin{aligned}
& \text { Direct: } \quad P_{l}=\mu_{i j k l} \frac{\partial S_{i j}}{\partial x_{k}}, \quad P_{l}=f_{i j k l} \frac{\partial T_{i j}}{\partial x_{k}} \\
& \text { Converse : } \quad T_{i j}=\mu_{i j k l} \frac{\partial E_{k}}{\partial x_{l}}, \quad S_{i j}=f_{i j k l} \frac{\partial E_{k}}{\partial x_{l}},
\end{aligned}
$$

where $P_{l}$ is the induced polarization, $\mu_{i j k l}$ is the flexoelectric coefficient, a fourth-rank tensor, $S_{i j}$ is the strain, and $x_{k}$ or $x_{l}$ is the axis; $T_{i j}$ is the induced stress, $f_{i j k l}$ is the converse flexoelectric coefficient associated with strain, and $E_{k}$ is the electric field.

The first phenomenological model of flexoelectricity in solid dielectrics was proposed by Kogan. ${ }^{5}$ However, in Kogan's original paper, he used "piezoelectric effect" to describe this unique inhomogeneous electro-mechanical coupling effect. By comparing it with an analogical phenomenon observed in liquid crystals, Indenbom suggested later that the term, "flexoelectric effect" should be adopted to describe this effect. ${ }^{6}$ It is one of the fundamental properties of crystalline dielectric materials and usually considered as a weak pseudo piezoelectric effect. ${ }^{7}$ In principle, flexoelectric effect is present universally in all 32 point groups and seven Curie groups of crystal structures without any symmetry constrains. ${ }^{8,9}$ Consequently, non-piezoelectric materials, such as cubic materials and isotropic materials, could still generate electric polarization under inhomogeneous mechanical field or yield mechanical strain under inhomogeneous electric field, through the direct and converse flexoelectric effect,

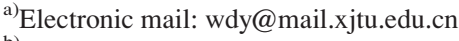

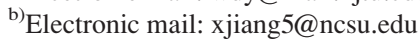

respectively. This unique property greatly enhances the potential of flexoelectric material as new and attractive sensing/actuating solid materials. ${ }^{10}$ However, theoretical value of flexoelectric constant is estimated to be in the order of $e / a\left(10^{-10} \mathrm{C} / \mathrm{m}\right)$, where $e$ is the electronic charge and $a$ is the crystal parameter. ${ }^{11}$ For most bulk materials, compared with piezoelectric constant, flexoelectric constant is so small that the related phenomena are always unperceivable in macro scale.

High level of direct flexoelectric effect was found in high permittivity perovskites such as Barium Strontium Titanate (BST), ${ }^{12}$ Barium Stannate Titanate (BTS), ${ }^{13}$ Barium Titanate (BT), ${ }^{14}$ Lead Magnesium Niobate (PMN), ${ }^{15}$ and Lead Zirconate Titanate (PZT). ${ }^{16}$ Intriguingly, the enhancement is four to five order larger than that of theoretical predictions. Moreover, PZT thin film ${ }^{17}$ and soft polymer polyvinylidene fluoride (PVDF) ${ }^{18}$ were also reported to have a large pure polarization when being bended. Recently, it was also reported that the scaling effect of direct flexoelectricity has great potential to be alternative of piezoelectricity for micro/nano devices. ${ }^{19-22}$

Although significant advances have been made in the research of direct flexoelectricity, the investigation on converse effect is rather limited. As proposed by Fu and Cross, ${ }^{23}$ one of the greatest challenges for converse flexoelectric measurement is the unavoidable influence from the simultaneous electrostriction effect. Conventionally, the influence of piezoelectricity can be readily excluded when non-piezoelectric material is selected for flexoelectric testing. However, both flexoelectric and electrostrictive effect can exist in all crystals without the symmetry limit. ${ }^{24,25}$ In one of our previous work, we demonstrated that the symmetry of flexoelectric coefficient is lower than electrostrictive constant $M_{i j k l}{ }^{9}$ This difference exists in low symmetry crystals but disappears in high symmetry crystals. For example, in a triclinic crystal, the number of non-zero independent components of 
$f_{i j k l}$ is 54 while 36 for $M_{i j k l}$. But for cubic or isotropic crystals, both $\mu_{i j k l}$ and $M_{i j k l}$ have the same non-zero components expressed as $f_{1111}, f_{1122}, f_{1212}$ and $M_{1111}, M_{1122}, M_{1212}$.

For most non-piezoelectric materials, $M_{i j k l}$ always plays a leading role for the contribution of strain. In terms of mathematics, a non-zero $\partial E_{i} / \partial x_{j}$ must be accompanied with $E_{i}^{2}$, thus $f_{1111}$ component coexists with $M_{1111}$ effect. In Fu's measurement, ${ }^{23}$ the flexoelectric strain is more than two orders smaller than electrostrictive strain, rendering it difficult to separate $f_{1111}$ from $M_{1111}$. However, $f_{1212}$ can exist alone when $\partial E_{1} / \partial x_{3} \neq 0, E_{1} E_{3}=0$ or can be a dominant contributor to a shear strain when $\partial E_{1} / \partial x_{3} \neq 0, E_{1} E_{3} \rightarrow 0$. In other words, a unidirectional electric field distribution is of highly significance. Here, we underline the word distribution, which stands for the orientation variation in a board range of material. In this study, we experimentally verify the pure converse flexoelectric effect by applying a directional electric field $E_{1}$, accompanied with a small $E_{3}$ component, as well as a large gradient $\partial E_{1} / \partial x_{3}$ so that the electrostrictive strain can be minimized in generating shear strain $S_{13}$.

The configuration to generate a directional electric field distribution is schematically shown in Fig. 1(a). Essentially, for a parallel plate capacitor, the electric field, as a continuous function, is perpendicular to the equipotential line and is reversely proportional to the effective distance between the two electrode plates. For the lateral plates of a truncated pyramid, considered as an unparalleled plate capacitor, the electric field $E_{1}$ is inhomogeneously distributed along the height direction $x_{3}$ due to the distance variation along direction $x_{1}$. The measurement setup was laid down on a float optical table (Newport, ATS, Irvine, CA) to eliminate the vibrational noise. The AC voltage was generated by a power amplifier (Trek, 2220, Lockport, NY) under the excitation from a function generator (Tectronix, AFG3101, Lake Mary, FL). As suggested in our previous work, ${ }^{9}$ the symmetry of the cubic materials permits only shear strain $S_{13}$, which is generated

(a)

Laser Vibrometer Reflection mirror

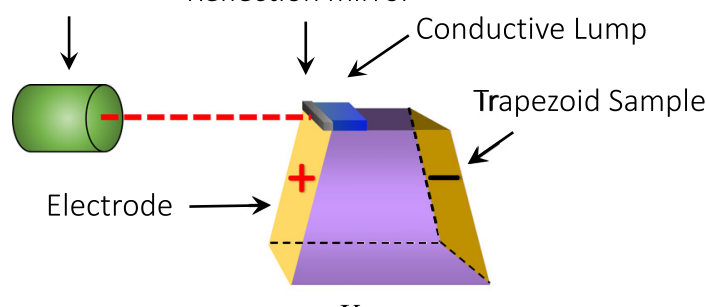

$V \cos w t$

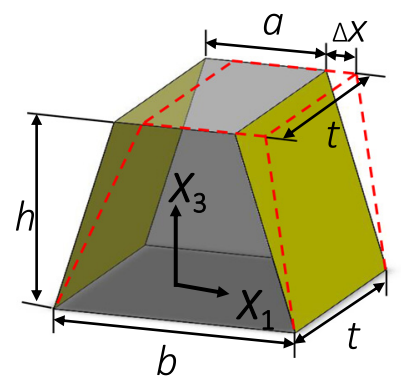

FIG. 1. (a) Diagram of sample assembly for converse flexoelectric measurement of the shear strain along $x_{1}$ direction generated by the electric field gradient along $x_{3}$ direction. (b) Schematic deformation of the trapezoid sample in the lateral mode. by $\partial E_{1} / \partial x_{3}$ due to the coupling of $f_{1212}$. To measure this shear strain, the bottom surface of the sample was clamped on the table and a mirror was attached to the top surface of the sample. The deformation along $x_{1}$ direction was measured using a high resolution $(<10 \mathrm{pm})$ laser vibrometer (Polytec, OFV-5000, Irvine, CA) and a lock-in amplifier (Stanford Research System, SR830, Sunnyvale, CA). Based on the measured shear deformation as illustrated in Fig. 1(b), the pure shear strain can be calculated as

$$
S_{5}=2 S_{13}=\frac{\Delta x}{h},
$$

where $\Delta x$ is the detected deformation and $h$ is the altitude of the sample.

The theoretical analysis for the electric field distribution in this non-parallel plate capacitor is shown in Fig. 2(a). When a voltage $V$ is applied across the sample, electric charge $Q$ will be accumulated on two surfaces. In nature, the electric field $(E)$ direction is dependent on the equipotential line $\varphi$. For the presented situation, both two side surfaces of the sample and the geometric center line are equipotential. The electric field would radiate along the tangential lines of the arc formed by two sides of the capacitor. It can be written as

$$
E_{r}=\frac{V}{r \theta},
$$

where $r$ is the radius of the scallop and $\theta$ is the angle of the scallop. Thus, the electric field is not homogeneous in light of the space curvature of the electric field line. In this case, an electric field gradient along the longitudinal direction $\left(x_{3}\right)$ is generated, as shown in Fig. 2(b). In addition, the angle between the electric field and $x_{1}$ axis changes from $-\theta / 2$ to $\theta / 2$ across the whole region due to the geometric relationship as illustrated in Fig. 2(c). Hence, the average electric field component for an arc with the angle of $\theta$ at the radius of $r$ in the transverse direction $\bar{E}_{1}$ can be obtained as

(a)

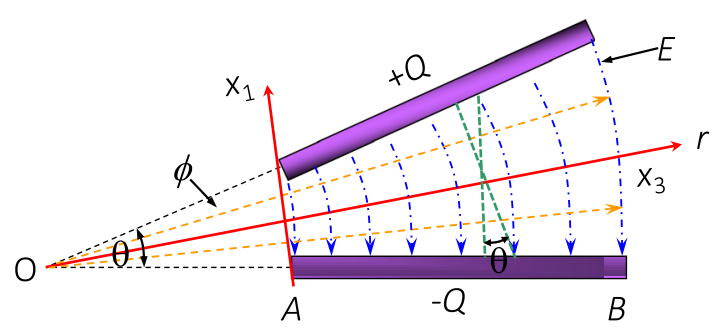

(b)

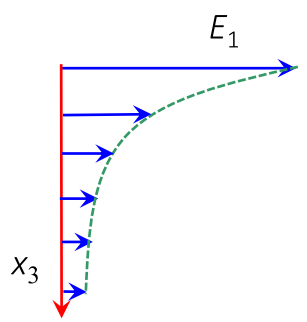

(c)

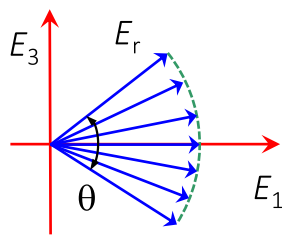

FIG. 2. Schematic view of a trapezoid sample with the voltage applied from side to side, (a) electric field line and equipotential distribution. $\mathrm{O}$ is the intersection point between the two extension lines of the slope side of the sample; (b) electric field gradient distribution; (c) electric field direction variation in arbitrary pitch arc $r$ position. 


$$
\int_{-\theta / 2}^{\theta / 2} E_{1} d \varphi=\int_{-\theta / 2}^{\theta / 2} E_{r} \cos \varphi d \varphi=\frac{\sin \theta / 2}{\theta / 2} E_{r},
$$

where $\varphi$ is the arbitrary vertex angle of the arc. In order to simply the calculation, the trapezoid sample was prepared with smooth side surfaces and rectangle top and bottom surfaces so that the average electric field $\bar{E}_{1}$ along the thickness gradient is

$$
\frac{\overline{\partial \overline{E_{1}}}}{\partial x_{3}}=\int_{a}^{b} \frac{\partial \overline{E_{1}}}{\partial x_{3}} d x=\frac{\sin ^{2} \theta / 2}{h(\theta / 2)}\left(\frac{V}{b}-\frac{V}{a}\right),
$$

where $a$ and $b$ are the side length of the top and bottom surface of the sample (Fig. 1(b)), respectively.

Equation (5) can be further simplified as Eq. (6) when $\theta$ is equal or lower than $\pi / 4$ (the coefficient equals to 0.95 )

$$
\frac{\overline{\partial E_{1}}}{\partial x_{3}}=\frac{1}{h}\left(\frac{V}{b}-\frac{V}{a}\right) .
$$

This simplified calculation was verified using the COMSOL Multiphysics (Fig. 3). A two dimensional trapezoid structure was considered in the simulation. The designated bottom and top lines have the length of $2.5 \mathrm{~mm}$ and $1.5 \mathrm{~mm}$, respectively. The height of the structure is $1 \mathrm{~mm}$. Material was chosen as the BST. Ground boundary condition was assigned to one of the two tilted side, while a DC voltage of $200 \mathrm{~V}$ was applied to the other tilted side. Electric field distribution along the horizontal direction $\left(E_{1}\right)$ can then be obtained. Further, numerical manipulation yields the electric field gradient along the vertical orientation $\left(\partial E_{1} / \partial x_{3}\right)$. The average magnitude was estimated to be $4.6 \times 10^{7} \mathrm{~V} / \mathrm{m}^{2}$, matching well with the theoretical calculation $\left(5.3 \times 10^{7} \mathrm{~V} / \mathrm{m}^{2}\right)$ using Eq. (6). There exist small singularity regions at the vertexes exhibiting high gradient concentrations. However, they would not make significant contribution to the averaged gradient value due to their small areas.

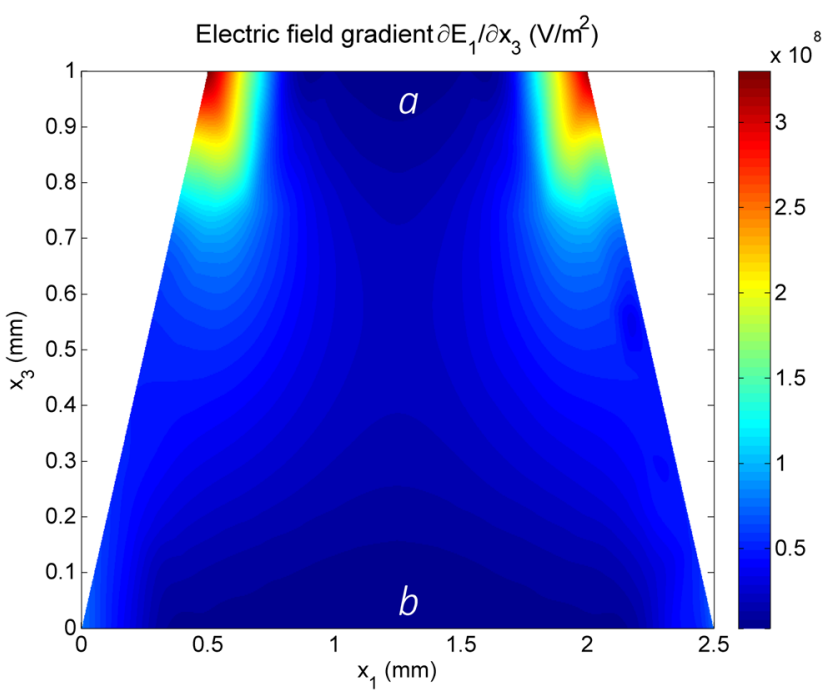

FIG. 3. Finite element simulation with COMSOL; areas with different color represent the magnitude of the calculated electric field gradient distribution within the trapezoid.
Moreover, the electric field component $E_{3}$ will, in principle, exist along with $E_{1}$ according to the above analysis. Nevertheless, the product of $E_{1} \bullet E_{3}$ is zero as interpreted in Eq. (7), so that it may not yield a shear strain coupled by electrostrictive constant $M_{1212}$.

$$
\int_{-\theta / 2}^{\theta / 2} E_{1} E_{3} d \varphi=\int_{-\theta / 2}^{\theta / 2} E_{r} \cos \varphi E_{r} \sin \varphi d \varphi=0 .
$$

This analysis theoretically ensures the feasibility of the above method for distinguishing flexoelectricity from electrostriction in converse flexoelectricity measurements.

Up to now, the maximum direct flexoelectric coefficient $(120 \mu \mathrm{C} / \mathrm{m})$ was observed in $\left(\mathrm{Ba}_{0.67} \mathrm{Sr}_{0.33}\right) \mathrm{TiO}_{3}$ (BST) ceramic at its paraelectric state (at temperature of $23{ }^{\circ} \mathrm{C}$, which is $2{ }^{\circ} \mathrm{C}$ above its $\mathrm{T}_{\mathrm{C}}$ ). ${ }^{12}$ The reason of this extraordinary enhancement is not clear yet. A large direct flexoelectric coefficient generally corresponds to a large converse coefficient. ${ }^{1}$ The aim of the present experiment is to identify the pure converse flexoelectric properties in the unpolarized BST ceramics. Three BST ceramic samples were prepared in specific shapes by a dicing saw (Disco, DAD320, Santa Clara, CA) and the dimensions are given in Table I. It is worthy to note that all three samples, including one sample with all rectangular surfaces (BST-I) and two samples with trapezoid shapes (BST-II and BST-III), were cut from the same raw material along one direction in order to exclude the orientation inhomogeneous of the material. Moreover, only the side surface of the sample is covered by electrode so that the influences from other directions do not exist. Electrode surfaces of all samples were coated with $200 \mathrm{~nm}$ thickness of nickel using a plating process (Transene, RTM Process, Danvers, MA). The sample BST-I was prepared as a reference where the electric field gradient is 0 .

The measured face displacement of samples BST-I, BST-II, and BST-III as a function of voltages ranged from $200 \mathrm{~V}$ to $700 \mathrm{~V}$ at $10 \mathrm{~Hz}$ are shown in Figs. 4(a)-4(c), respectively. A visible $20 \mathrm{~Hz}$ response was found in all of the samples, displaying with a quadratic regulation in plot. In principle, in this experiment, irrespective the symmetry of the sample, all the possible contributions to shear strain $S_{13}$ can be expressed as

$$
\begin{aligned}
S_{13}= & d_{113} E_{1} \cos \omega t+f_{1212} \frac{\partial E_{1}}{\partial x_{3}} \cos \omega t \\
& +M_{1212} E_{1} E_{2} \cos \omega t \cos \omega t \\
= & S_{13}(P)+S_{13}(F)+S_{13}(M),
\end{aligned}
$$

where $d_{113}$ is the shear piezoelectric constant and $\omega$ is the driven frequency. Hereafter, the piezoelectric, flexoelectric, and electrostriction contribution is labeled as $P, F$, and $M$, respectively. As interpreted in Eq. (8), the origin of $2 \omega$-signal is only associated with $M$, while the response with frequency of $\omega$ is yielded by $P$ and $F$. For the sample shown in Fig. 4(a), $M_{1212}$ contribution does not exist due to absence of $E_{3}$. However, the clamped condition of bottom cannot restrict the oscillation of the top of the sample. The electric field in transverse direction would yield the direct displacement reflected in the mirror due to the coupling of the $M_{1111}$, thus to cause 
TABLE I. Dimension of BST samples.

\begin{tabular}{lccccc}
\hline \hline Sample & $\begin{array}{c}\text { Cross section shape } \\
\left(x_{1}-x_{3} \text { plane }\right)\end{array}$ & $\begin{array}{c}\text { Vertex angle } \\
\theta(\text { degree })\end{array}$ & $\begin{array}{c}\text { Top length } \\
x_{1} a(\mathrm{~mm})\end{array}$ & $\begin{array}{c}\text { Bottom length } \\
x_{1} b(\mathrm{~mm})\end{array}$ & $\begin{array}{c}\text { Height } \\
x_{3} h(\mathrm{~mm})\end{array}$ \\
\hline BST I & Rectangle & 90 & 3.64 & 3.64 & 3.03 \\
BST II & Trapezoid & 62 & 1.83 & 5.05 & 3.03 \\
BST III & Trapezoid & 46 & 1.83 & 7.55 & 3.03 \\
\hline
\end{tabular}

$2 \omega$-response, as seen in the measured second harmonic results. In addition, a non-zero first harmonic response of the BST-I sample implies the existence of piezoelectric effect in BST, which contradicts the fact that the symmetry of BST material in paraelectric phase permits no macro piezoelectricity. Nevertheless, when the temperature is very close to the Curie temperature, ferroelectricity may exist due to local nano domains ${ }^{26}$ which may be one possible reason for the existence of piezoelectric responses in BST samples.

On the other side, in Fu's experiment, ${ }^{23}$ the driving frequency of the power amplifier was $400 \mathrm{~Hz}$. It is possible that the output waveform of the power amplifier was distorted, and hence, was not a standard cosine function at such a high driving frequency. In this case, the first harmonic signal is unavoidably influenced by the coupling of electrostrictive effect. As interpreted by Fu et al., the small DC voltage shifts $\tilde{n}$ generated by the power amplifier, resulting in a first harmonic response $2 M_{i j k l} \tilde{n} V_{1} \cos \omega t$. In our experiment, the driven frequency was only $10 \mathrm{~Hz}$ and there was no detected $10 \mathrm{~Hz}$ background and electromagnetic noise, as shown in Figs. S1 and S2, respectively. Moreover, the driving outputs of the power amplifier were cosine waveforms as demonstrated in supplementary material Figs. S3, S4, and S5, so that $M$ is proportional to the square of the electric field and would not contribute to the first harmonic response. ${ }^{29}$ In other words, the first harmonic value $\Delta x(\mathrm{~V})$, in principle, does not encompass the influence from $M$ and thus, can be used to calculate the contribution from $P$ and $F$.

For rectangular sample BST-I, $\Delta x(V)$ is only derived from the piezoelectric displacement contribution $\mathrm{P}$

$$
\Delta x(V)=2 \int d_{113} E_{1}(z) d z=\frac{2 d_{113} h}{W} V,
$$

where $W$ is the width and $\mathrm{z}$ is the arbitrary position in the height direction.

As for the trapezoid samples II and III, $\Delta x(V)$ is expressed as

$$
\begin{aligned}
\Delta x(V) & =2 \int\left(d_{113} E_{1}(z)+f_{1212} \frac{\partial E_{1}(z)}{\partial z}\right) d z \\
& =2 \int_{0}^{h} d_{113} \frac{V}{a+2(h-z) \cot \theta} d z+2 \int_{0}^{h} f_{1212} \frac{\partial E_{1}}{\partial z} d z,
\end{aligned}
$$

where

$$
\int_{0}^{h} d_{113} \frac{V}{a+2(h-z) \cot \theta} d z=\frac{\tan \theta}{2} d_{113} \ln \frac{b}{a} V
$$
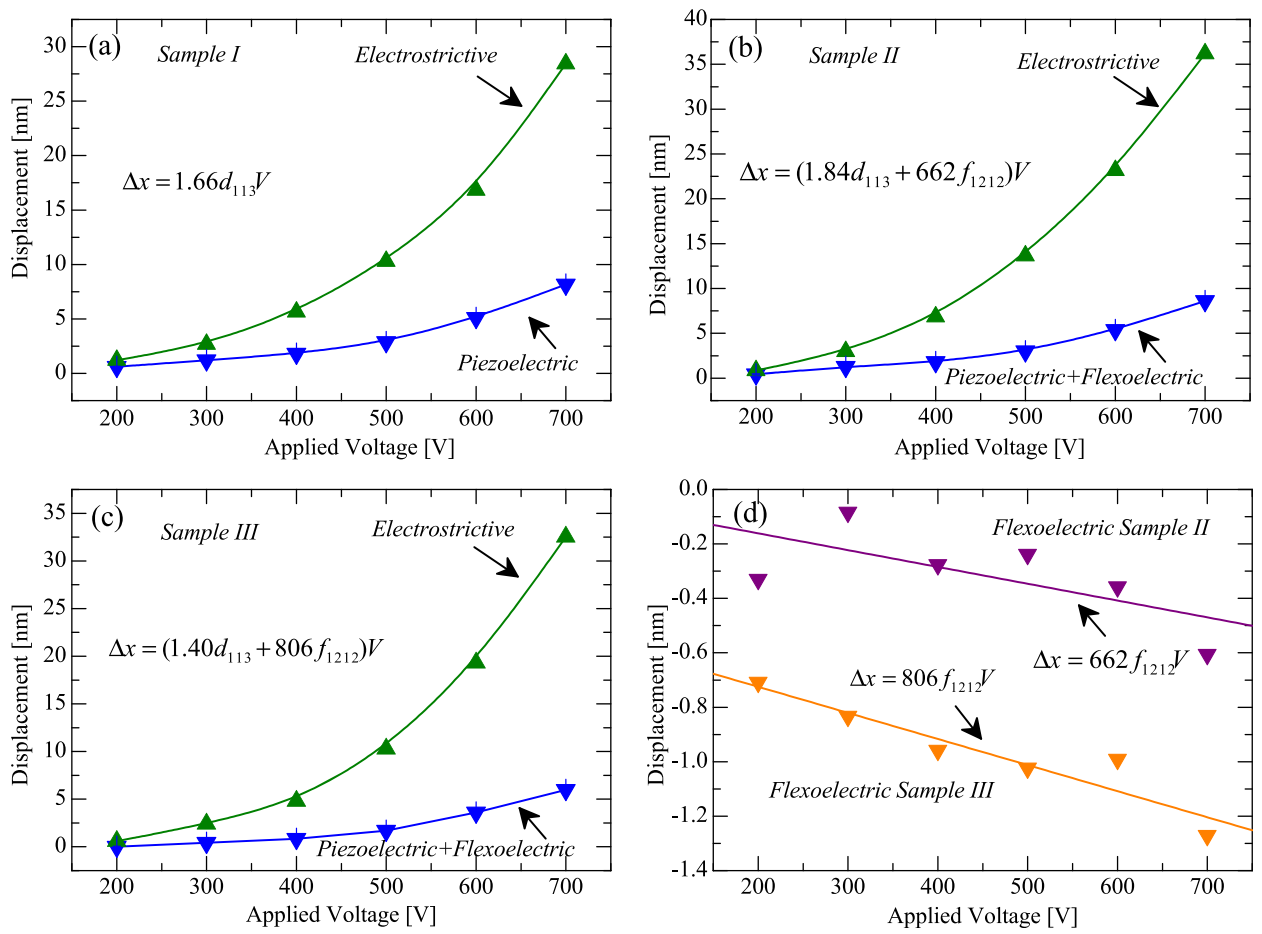

FIG. 4. Face displacement measured using a laser vibrometer as a function of voltage in room temperature for BST samples: (a) rectangle; (b) trapezoid $62^{\circ}$; and (c) trapezoid $46^{\circ}$. The green points are the second harmonic displacement associated with the electrostrictive effect. The blue points stand for the displacement associated with the piezoelectric and flexoelectric effect. The solid line is a guide to the eye. (d) Pure converse flexoelectric coefficient $f_{1212}$ contribution extracted from the displacement subtraction in BST-II and BST-III as a function of voltage, respectively. The solid lines are the fitting slope of face displacement vs. voltage induced by net converse flexoelectric effect. 


$$
\int_{0}^{h} f_{1212} \frac{\partial E_{1}}{\partial z} d z=\left.f_{1212} E_{1}\right|_{0} ^{h}=f_{1212} \frac{\sin \theta / 2}{\theta / 2} V\left(\frac{1}{a}-\frac{1}{b}\right) .
$$

It is noticed that $P$ is proportional with the angle and the bottom/top dimension ratio while $F$ is reciprocal proportional with the dimension. This calculation suggests that $F$ likely plays a dominant role when scaling down the dimension. Thus, we can calculate the $P$ and $F$ proportions in each sample with the relevant sample parameters given in Table I. The displacement information including first harmonic under different voltage direction and second harmonic of samples II and III are represented in Figs. 4(b) and 4(c). The contributions from $P$ and $F$ are given in the legends. We mention that all types of samples were prepared with a counterpart and each sample was tested for three times under the same AC voltage. The repeatability of the displacement, measured by the laser vibrometer, is observed well due to the minimization of vibrational noise by a floating optical table and the absence of mechanical impact from electric equipment (supplementary material Table SI).

Based on the above measurement results, pure $F$ in BST-II and BST-III ceramics can be extracted by subtracting the associated piezoelectric displacement contribution (Fig. 4(d)). This calculation is based on an assumption that all the material constants in Eq. (8) are the same for all three samples. The identical sample preparation process ensures the homogeneity of the sample. Linear fitting of the $F$ induced displacement yields the converse flexoelectric coefficient $f_{1212}$ to be $9.3 \times 10^{-16} \mathrm{~m}^{2} / \mathrm{V}$ and $1.18 \times 10^{-15} \mathrm{~m}^{2} / \mathrm{V}$ for sample II and sample III, respectively. The less common used unit $\mathrm{m}^{2} / \mathrm{V}$ can be converted to the nominal unit $\mathrm{C} / \mathrm{m}$ by removing the factor of elastic constant. Generally, the elastic compliance constant $s_{1212}$ of BST ceramics is around $8.5 \times 10^{-12} \mathrm{~m}^{2} / \mathrm{N},{ }^{27}$ by which the stress related flexoelectric constant $\mu_{1212}$ is estimated to be $110 \mu \mathrm{C} / \mathrm{m}$ (sample II) and $138 \mu \mathrm{C} / \mathrm{m}$ (sample III). We note that the minus slope of the high linearity line stands for the deformation direction induced by electric field gradient, which is different from the piezoelectric deformation direction for this material.

This converse flexoelectric coefficient is similar to the direct result $\mu_{1111}(120 \mu \mathrm{C} / \mathrm{m})$ observed in the same materials. ${ }^{28}$ As demonstrated in previous work, the relationship among the non-zero dependent components of the flexoelectric coefficients in isotropic crystals is expressed as $\mu_{1212}=1 / 2\left(\mu_{1111}-\mu_{1122}\right){ }^{9}$ In this case, the value of the $\mu_{1212}$ is half of the subtraction between $\mu_{1111}$ and $\mu_{1122}$ due to the high symmetry of the materials. In rigid ceramic materials, generally, stretch along one dimension is accompanied with compression in another two dimensions. Therefore, the sign of $\mu_{1122}$ should be opposite to that of $\mu_{1111}$, just like the sign of elastic coefficient $s_{11}$ is opposite to that of $s_{12}$ and the sign of $d_{33}$ is opposite to that of $d_{31}$. Based on above analysis, the theoretical prediction of $\mu_{1212}$ in the BST is estimated to be $110 \mu \mathrm{C} / \mathrm{m}$, which is comparable with the direct $\mu_{1111}$ $(120 \mu \mathrm{C} / \mathrm{m})^{26}$ and $\mu_{1122}(100 \mu \mathrm{C} / \mathrm{m}){ }^{12}$ Our experiment first observed the shear flexoelectric data being consistent with the theoretical prediction.
In conclusion, converse flexoelectric effect of BST was investigated by generating a unidirectional electric field distribution along height direction of trapezoid samples. The present study clearly demonstrated the linear relationship between the electric field gradient and shear strain. The converse flexoelectric coefficient $\mu_{1212}$ is estimated to be 124 $\pm 14 \mu \mathrm{C} / \mathrm{m}$, being in good agreement with the theoretical prediction. This result could benefit the development of electromechanical devices based on the flexoelectric effect. For a properly designed trapezoid sample, a small electric field can generate a large electric field gradient so that a visible shear deformation may be dominated by converse flexoelectric effect rather than the piezoelectric effect, especially, when the sample dimension scales down to the micro/nano level.

L.S. acknowledges extensive discussions with Dr. Li Jin. This work was supported by the International Science \& Technology Cooperation Program of China (2013DFR50470) and "111" Project (B14040), and in part by National Science Foundation under Grant No. CMMI-1068345. L.S. thanks the support from Xi'an Jiaotong University.

${ }^{1}$ P. V. Yudin and A. K. Tagantsev, Nanotechnology 24, 432001 (2013).

${ }^{2}$ X. Jiang, W. Huang, and S. Zhang, Nano Energy 2, 1079 (2013).

${ }^{3}$ P. Zubko, G. Catalan, and A. K. Tagantsev, Annu. Rev. Mater. Res. 43, 387 (2013).

${ }^{4}$ L. E. Cross, J. Mater. Sci. 41, 53 (2006).

${ }^{5}$ S. M. Kogan, Sov. Phys. Solid State 5, 2069 (1964).

${ }^{6}$ V. L. Indenbom, E. B. Loginov, and M. A. Osipov, Sov. Phys. Crystallogr. 26, 656 (1981).

${ }^{7}$ R. Resta, Phys. Rev. Lett. 105, 127601 (2010).

${ }^{8}$ H. Quang and Q. He, Proc. R. Soc. London, Ser. A 467, 2369 (2011).

${ }^{9}$ L. Shu, X. Wei, T. Pang, X. Yao, and C. Wang, J. Appl. Phys. 110, 104106 (2011).

${ }^{10}$ W. Huang, X. Yan, S. Kwon, S. Zhang, F. Yuan, and X. Jiang, Appl. Phys. Lett. 101, 252903 (2012).

${ }^{11}$ A. K. Tagantsev, Sov. Phys. JETP 61, 1246 (1985).

${ }^{12}$ W. Ma and L. E. Cross, Appl. Phys. Lett. 81, 3440 (2002).

${ }^{13}$ L. Shu, X. Wei, L. Jin, Y. Li, H. Wang, and X. Yao, Appl. Phys. Lett. 102, 152904 (2013).

${ }^{14}$ W. Ma and L. E. Cross, Appl. Phys. Lett. 88, 232902 (2006).

${ }^{15}$ W. Ma and L. E. Cross, Appl. Phys. Lett. 79, 4420 (2001).

${ }^{16}$ W. Ma and L. E. Cross, Appl. Phys. Lett. 86, 072905 (2005).

${ }^{17}$ Z. Wang, X. Zhang, X. Wang, W. Yue, J. Li, J. Miao, and W. Zhu, Adv. Funct. Mater. 23, 124 (2013).

${ }^{18}$ S. Baskaran, X. He, Q. Chen, and J. Y. Fu, Appl. Phys. Lett. 98, 242901 (2011).

${ }^{19}$ M. Majdoub, P. Sharma, and T. Cagin, Phys. Rev. B 77, 125424 (2008).

${ }^{20}$ W. Huang, K. Kim, S. J. Zhang, F. G. Yuan, and X. N. Jiang, Phys. Status Solidi RRL 5, 350 (2011).

${ }^{21}$ J. Y. Fu, W. Zhu, N. Li, N. B. Smith, and L. Cross, Appl. Phys. Lett 91, 182910 (2007).

${ }^{22}$ J. Y. Fu and L. E. Cross, Appl. Phys. Lett. 91, 162903 (2007).

${ }^{23}$ J. Y. Fu, W. Zhu, N. Li, and L. E. Cross, J. Appl. Phys. 100, 024112 (2006).

${ }^{24}$ F. Li, L. Jin, Z. Xu, D. Wang, and S. Zhang, Appl. Phys. Lett. 102, 152910 (2013).

${ }^{25}$ F. Li, L. Jin, Z. Xu, and S. Zhang, Appl. Phys. Rev. 1, 011103 (2014).

${ }^{26}$ M. Roth, E. M. E. Dulkin, P. Gemeiner, and B. Dkhi, Phys. Rev. Lett. 98, 265701 (2007).

${ }^{27}$ V. B. Shirokov, Y. I. Yuzyuk, V. V. Kalinchuk, and V. V. Lemanov, Phys. Solid State 55, 773 (2013).

${ }^{28}$ W. Zhu, J. Y. Fu, N. Li, and L. E. Cross, Appl. Phys. Lett. 89, 192904 (2006).

${ }^{29}$ See supplementary material at http://dx.doi.org/10.1063/1.4882060 for more details on the experimental testing signal to noise ratio, repeated testing results and calculation details. 